\title{
Natural assembly of platelet lysate-loaded nanocarriers into enriched 3D hydrogels for cartilage regeneration
}

\author{
Vítor E. Santo, Elena G. Popa, João F. Mano, Manuela E. Gomes, Rui L. Reis* \\ 3B's Research Group - Biomaterials, Biodegradables and Biomimetics, University of Minho, Headquarters of the European Institute of Excellence on Tissue Engineering \\ and Regenerative Medicine, AvePark, 4806-909 Taipas, Guimarães, Portugal \\ ICVS/3B's - PT Government Associate Laboratory, Braga/Guimarães, Portugal
}

\section{A R T I C L E I N F O}

\section{Article history:}

Received 22 October 2014

Received in revised form 4 February 2015

Accepted 11 March 2015

Available online $\mathrm{xxxx}$

\section{Keywords:}

Platelet lysates

Nanoparticles

Tissue engineering

Self-assembly

Hydrogel

\begin{abstract}
A B S T R A C T
The role of Platelet Lysates (PLs) as a source of growth factors (GFs) and as main element of threedimensional (3D) hydrogels has been previously described. However, the resulting hydrogels usually suffer from high degree of contraction, limiting their usefulness. This work describes the development of a stable biomimetic 3D hydrogel structure based on PLs, through the spontaneous assembling of a high concentration of chitosan-chondroitin sulfate nanoparticles ( $\mathrm{CH} / \mathrm{CS}$ NPs) with PLs loaded by adsorption. The interactions between the NPs and the lysates resemble the ones observed in the extracellular matrix (ECM) native environment between glycosaminoglycans and ECM proteins. In vitro release studies were carried out focusing on the quantification of PDGF-BB and TGF- $\beta 1$ GFs. Human adipose derived stem cells (hASCs) were entrapped in these 3D hydrogels and cultured in vitro under chondrogenic stimulus, in order to assess their potential use for cartilage regeneration. Histological, immunohistological and gene expression analysis demonstrated that the PL-assembled constructs entrapping hASCs exhibited results similar to the positive control (hASCS cultured in pellets), concerning the levels of collagen II expression and immunolocalization of collagen type I and II and aggrecan. Moreover, the deposition of new cartilage ECM was detected by alcian blue and safranin-O positive stainings. This work demonstrates the potential of PLs to act simultaneously as a source/carrier of GFs and as a 3D structure of support, through the application of a "bottom-up" approach involving the assembly of NPs, resulting in an enriched construct for cartilage regeneration applications.
\end{abstract}

(c) 2015 Acta Materialia Inc. Published by Elsevier Ltd. All rights reserved.

\section{Introduction}

Cartilage is a predominantly avascular tissue characterized by sparsely distributed chondrocytes embedded within a dense extracellular matrix (ECM), which is mainly composed of type II collagen and proteoglycans, particularly chondroitin sulfate. Due to its limited ability to self-repair, cartilage is an ideal candidate for tissue engineering (TE) [1-5]. During chondrogenic differentiation, cells prefer a three-dimensional (3D) environment, provided in the simplest strategy by the cells themselves, referred as "micromass pellet culture" $[3,6]$ which attempts to mimic the mesenchymal condensation that happens during embryonic cartilage development $[2,7]$. However in the in vivo scenario, the direct implantation

\footnotetext{
* Corresponding author at: 3B's Research Group - Biomaterials, Biodegradables and Biomimetics, University of Minho, Headquarters of the European Institute of Excellence on Tissue Engineering and Regenerative Medicine, AvePark, 4806-909 Taipas, Guimarães, Portugal.

E-mail address: rgreis@dep.uminho.pt (R.L. Reis).
}

of the cells is usually not enough to obtain a successful defect regeneration, being required the use of a suitable biomaterial that acts as a template for cell proliferation and differentiation, providing also a temporary mechanical support to withstand the tissue regeneration requirements [3].

Hydrogels can homogeneously encapsulate cells in a 3D environment with high water content and mechanical properties reminiscent of cartilage tissue, maintaining a rounded cell morphology which may elicit a chondrocytic phenotype $[2,8,9]$. Hydrogels based on biopolymers mimic many features of the ECM and thus have the potential to direct the migration, growth and organization of cells during tissue regeneration [10]. A wide range of natural and synthetic polymeric materials have been investigated as scaffolding for cartilage repair, including alginate $[11,12]$, agarose [13,14], gelatin [15], hyaluronic acid [16,17], gellan gum [18,19], carrageenan [20,21], and fibrin [22,23], aiming at supporting the in vitro and in vivo chondrogenic differentiation of Mesenchymal Stem Cells (MSCs) [2,3]. 
Stem cells, and particularly MSCs, have generated significant interest in cartilage TE as a cell source alternative to autologous chondrocytes $[2,24]$. In fact, the number of chondrocytes that can be isolated primarily from the native cartilage is limited [24] and they exhibit a very limited proliferation capacity, which draws a serious limitation to their expansion in culture [25]. Moreover, the relative instability of their phenotype in cell culture and continuous dedifferentiation remain a challenge $[9,25]$. Thus, obtaining multipotent cells which are capable of differentiating toward the chondrogenic lineage [3] from adult tissues such as bone marrow or adipose tissue is currently considered an advantageous procedure. While bone marrow provides the most universal source of MSCs, adipose tissue offers a much more abundant and easily accessible pool of MSCs [26]. Adipose derived stem cells (ASCs) have been shown to be able to differentiate toward the chondrogenic lineage in high density micromass cultures, polymeric scaffolds and hydrogels [2,6,27-29].

The combination of the three fundamental tools, namely cells, engineering materials and signaling molecules constitutes the triad of TE aiming to recreate the functional tissue [30]. Growth factors (GFs) can enhance cartilage formation by different mechanisms, including through recruitment of chondrogenic cells, enhancement of cell proliferation and of cartilage matrix synthesis [10]. The use of platelet concentrates as an alternative approach to the release of multiple GFs for therapeutic applications is a very exciting area of research [30]. Platelets contain a wide range of cytokines such as the isoforms of Insulin Growth Factor (IGF), Platelet Derived
Growth Factor (PDGF), Transforming Growth Factor (TGF), among others [31]. Platelet derived products are termed Platelet Lysates (PLs) or Platelet Rich Plasma (PRP) and have been studied and used since the 1970s [32]. They also have been suggested to possess impact in the improvement of articular cartilage disorders [3]. Different authors $[33,34]$ have demonstrated that PLs also stimulate the proliferation of chondrocytes. However, controversial results are published on their influence over the chondrogenic differentiation and cartilage matrix accumulation [3,35]. PRP has been used not only as a cytokine supplement or GF source [36] but also as the main material to produce 3D scaffolds/hydrogels [10]. However, their main disadvantage is the increasing instability and solubility over time, in vitro and in vivo. The hydrogels tend to considerably shrink, especially when seeded with cells, which represents an important drawback for application as cell encapsulation systems [2,37].

In this study we report the development of a novel hydrogel system based on the assembly of chitosan-chondroitin sulfate nanoparticles (CH/CS NPs) carrying platelet lysates (PLs). The chitosan/chondroitin sulfate nanoparticles were developed in previous studies [38], where it was demonstrated that these NPs were able to support the sustained release of proteins. The design of the system proposed herein is based on the fact that when used at high concentrations, the PL-releasing NPs spontaneously assemble in a simple and quick mode to form a 3D hydrogel, capable of entrapping cells (Fig. 1). Human ASCs (hASCs) were cultured onto these constructs for up to 28 days and the in vitro chondrogenic

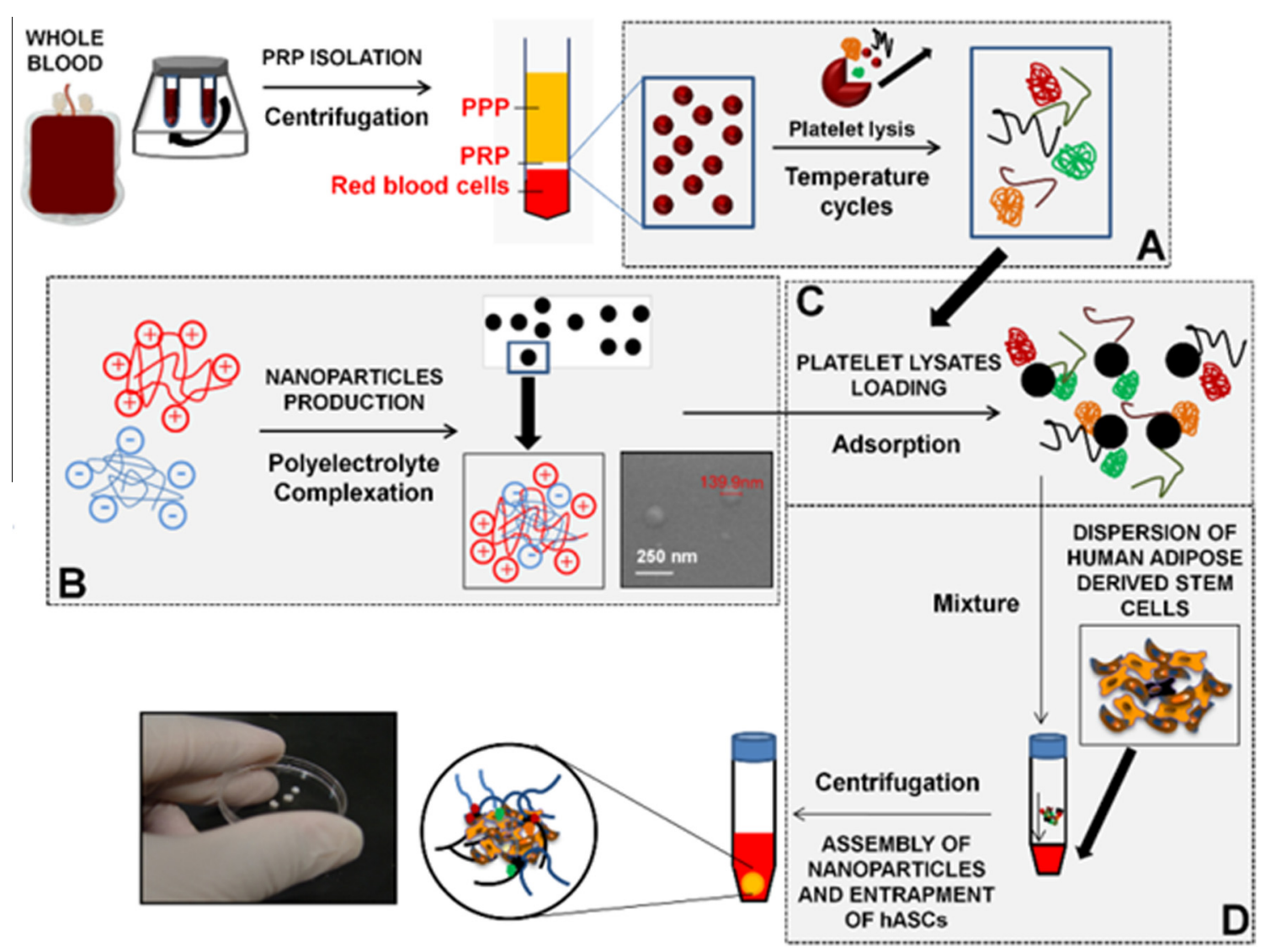

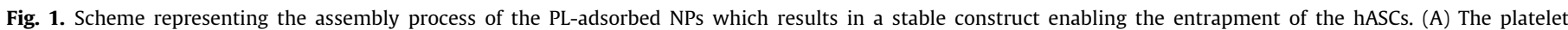

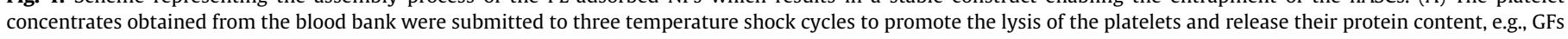

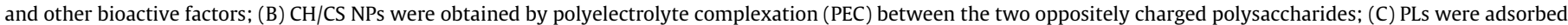

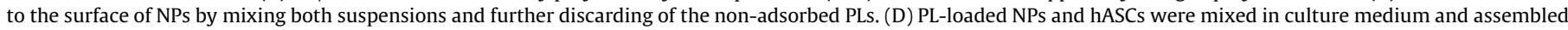
together by a centrifugation step, obtaining a hydrogel-like construct with the hASCs entrapped in the network of CH/CS NPs, linked together by the adsorbed PLs. 
differentiation of the hASCs and possible formation of a cartilaginous ECM were quantitatively and qualitatively assessed by various techniques.

\section{Materials and methods}

\subsection{Materials}

Low molecular weight (116 kDa) chitosan (CH) (Ref: 448869), chondroitin sulfate (CS) (C9819), phosphate buffered saline tablets (PBS), Dulbecco's modified Eagle's medium (DMEM) low glucose, Dulbecco's modified Eagle's medium without phenol red, sodium bicarbonate and dexamethasone were purchased from Sigma Aldrich (Germany). Fetal bovine serum (FBS) was purchased from Biochrom AG (Germany) and antibiotic-antimycotic (A/B) solution from Gibco (Spain). (3-(4,5-dimethylthiazol-2-yl)-5-(3carboxymethoxyphenyl)-2-(4-sulfophenyl)-2H-tetrazolium) MTS reagent was purchased from Promega (G5421, UK). Chitosan was further purified by the precipitation procedure, all the other chemicals were used with no further purification. After chitosan purification, the final deacetylation degree was 75-85\%. ELISA kits for the detection of human PDGF-BB and TGF- $\beta 1$ were purchased from R\&D Systems.

\subsection{Platelet lysate preparation}

PLs were obtained from different platelet collections performed at Instituto Português do Sangue (IPS, Porto, Portugal), under a previously established cooperation protocol. The components were obtained using the Trima Accel ${ }^{\circledR}$ Automated Blood Collection System. All the platelet products were biologically qualified according to the portuguese legislation. The platelet count was performed at the IPS using the COULTER ${ }^{\circledR}$ LH 750 Hematology Analyzer and the sample volume was adjusted to 1 million platelets per $\mu \mathrm{L}$. The samples were transferred to the laboratory within $24 \mathrm{~h}$ after collection and immediately submitted to three repeated temperature cycles (frozen with liquid nitrogen at $-196^{\circ} \mathrm{C}$ and heated at $37^{\circ} \mathrm{C}$ ) [39], followed by a centrifugation step at $1400 \mathrm{~g}$ for $10 \mathrm{~min}$, to eliminate the platelet debris. The lysates were then frozen at $-20^{\circ} \mathrm{C}$ until further use. The platelets remaining were eliminated by centrifugation at $1400 \mathrm{~g}$ for $10 \mathrm{~min}$.

\subsection{Preparation of the empty and platelet lysate-loaded nanoparticles}

Chitosan/chondroitin sulfate nanoparticles (CH/CS NPs) were prepared as described previously [38]. For further experimental details, check Supplementary Information (SI). To prepare the PL-loaded $\mathrm{CH} / \mathrm{CS}$ NPs, the PLs (mixed from three different donors to reduce the effect of batch-to-batch variability) were entrapped by adsorption on the structure to the empty $\mathrm{CH} / \mathrm{CS}$ NPs, as follows: approximately $12 \mathrm{mg}$ of empty $\mathrm{CH} / \mathrm{CS} 1 / 1 \mathrm{NPs}$ was incubated with $5 \mathrm{~mL}$ of PLs during $45 \mathrm{~min}$. After that, the particles were allowed to sediment for $30 \mathrm{~min}$ and the excess of PLs was removed. Following a quick spin-down, the supernatant was discarded and the PL-loaded NPs were isolated.

\section{4. hASC isolation}

Human subcutaneous adipose tissue samples were obtained from elective lipoaspiration procedures performed on women with ages between 35 and 50 years under a protocol previously established with the Department of Plastic Surgery of Hospital da Prelada in Porto, Portugal. All the samples were processed within $24 \mathrm{~h}$ after the lipoaspiration procedure. Human ASCs were enzymatically isolated from subcutaneous adipose tissue as previously described $[40,41]$. The complete isolation protocol is available as SI.

\subsection{Entrapment of hASCs within the assembled PL-loaded NPS}

To obtain each sample/construct, approximately $1 \mathrm{mg}$ of PLloaded NPs previously prepared as described above, was resuspended in $5 \mathrm{~mL}$ of DMEM medium. Afterward, the NPs were gently mixed with 1 million of hASCs suspended in $2 \mathrm{~mL}$ of DMEM in order to obtain a homogeneous dispersion of cells/NPs. The mixture was then centrifuged at $1500 \mathrm{rpm}$ for $10 \mathrm{~min}$ and a pellet-like hydrogel was formed. The same procedure was carried out using empty NPs, attempting to obtain a similar hydrogel/structure. Pellets built only with hASCs ( 1 million cells per construct) were also prepared to be used as controls. The cell-constructs (with either empty or PL-loaded NPs) and the hASC pellets were then cultured in vitro in chondrogenic medium composed of DMEM supplemented with $1 \mathrm{mM}$ dexamethasone, $0.1 \mathrm{M}$ sodium pyruvate (P4562 Sigma, Germany), 17 mM L-ascorbic acid (A4544 Sigma, Germany), 35 mM L-proline (P5607 Sigma, Germany), ITS Liquid Media Supplement (I2521-insulin-transferrin-selenium-liquid media supplement, Sigma, Germany), 1\% antibiotic and 10\% FBS, for periods $1,7,14,21$ and 28 days, in a humidified atmosphere at $37{ }^{\circ} \mathrm{C}$, containing $5 \% \mathrm{CO}_{2}$. The pellet cultures were also supplemented with $10 \mathrm{ng} / \mathrm{mL}$ of TGF- $\beta 1$. The culture medium was changed every 3-4 days until the end of the experiment.

\subsection{In vitro release studies}

Each study sample/construct was formed from approximately $1 \mathrm{mg}$ of PL-loaded NPs resuspended in PBS and centrifuged at $1500 \mathrm{rpm}$ for $10 \mathrm{~min}$ to form a pellet-like hydrogel. The same procedure was applied using empty NPs, attempting to obtain a similar hydrogel to be used as control. In vitro release studies of GFs known to be present in significant amounts in the PLs, namely PDGF-BB and TGF- $\beta 1$, were performed, following the protocol described in SI.

\subsection{Morphological characterization of the constructs - Scanning Electron Microscopy}

The morphology of the assembled NP-PL hydrogels with entrapped cells was observed by SEM. Energy dispersive spectroscopy (EDS) (Pegasus X4 M) was used to perform elemental analysis on the materials. For further details, check SI.

\subsection{Cellular viability assay}

Cell viability was assessed after 1, 7, 14, 21 and 28 days, using the MTS test and the live/dead assay (calcein AM/propidium iodide (PI) staining). Complete protocols are available as SI.

\subsection{Cell proliferation by DNA quantification}

Human ASC proliferation on the constructs was determined using a fluorimetric dsDNA quantification kit (PicoGreen, Molecular Probes, Invitrogen, USA), according to the manufacturer's instructions as described in SI.

\subsection{Histological analysis}

Constructs were harvested at the specified time points and fixed with $4 \%$ paraformaldehyde. The specimens were included in paraffin and tissue sections of $5 \mu \mathrm{m}$ of thickness were obtained using a microtome (Microm HM355S, Thermo Scientific, Germany), and mounted in a micro-slide glass. 
Hematoxylin \& Eosin (H\&E), alcian blue and safranin O stainings were then performed, following standard histological procedures using the automatic stainer (Microm HMS 740, Thermo Scientific, Germany) as described in SI and scored according to the Bern scoring system [42].

\subsection{Immunohistochemistry}

Immunostaining for collagen type I, type II and aggrecan antibodies was performed to assess the formation of cartilaginous-like ECM. Complete protocol is available in SI.

\subsection{Real time reverse transcriptase polymerase chain reaction}

The expression of mRNA for the genes of interest, usually used to characterize the chondrogenic phenotype, namely collagen I and collagen II, was measured by real time RT-PCR analysis. Relative quantification was calculated using $2^{-\Delta \Delta \mathrm{Ct}}$ method for the constructs [43]. Experimental details are described in SI.

\subsection{Statistical analysis}

All the experiments were performed with at least three replicates. The unpaired $t$-test was used for comparisons between two groups only. The one-way ANOVA, followed by the Tukey's post hoc, was used for comparisons between more than two groups. $p<0.05$ was considered significant. Data are shown as mean \pm standard deviation of the means.

\section{Results}

Fig. 2 displays morphological characterization of the assembled hydrogels and the release profiles of two of the most important GFs present in the PLs. PDGF-BB and TGF- $\beta 1$ are some of the isoforms present in higher concentration in the protein cocktail. Fig. $2 \mathrm{~A}$ shows a representative image of the formed hydrogel, with $6 \mathrm{~mm}$ of height. Regarding the release studies, a strong burst effect for both GFs was observed during the first day of delivery, with a visible stabilization of the release rate for the following days of the experimental period. In fact, after one day of release, around $2750 \mathrm{pg}$ and $2200 \mathrm{pg}$ of PDGF-BB and TGF- $\beta 1$ were released and these account for $92 \%$ and $80 \%$ of the total release for each GF after seven days of experiment, respectively. After 7 days, the delivery was minimal and/or below the detection limit of the ELISA kits.

Assembled PL-loaded NP constructs were prepared with approximately 4.75 and $4 \mathrm{ng} / \mathrm{mL}$ of PDGF-BB and TGF- $\beta 1$ per hydrogel, respectively. By comparing these initial protein loadings with the detected GF release profiles, a GF cumulative delivery of approximately $61 \%$ and $70 \%$ was attained for PDGF-BB and TGF$\beta 1$, respectively.

Although SEM is not the best technique to characterize the morphological structure of hydrogels, we can observe that PL-loaded hydrogels presented a more homogeneous surface (Fig. 2C) than the hydrogels resultant from the assembly of empty NPs (Fig. 2B). The EDS analysis was performed in different areas of the material represented in Fig. 2C. At this magnification, it was possible to observe differences on the surface topography of the sample, with smoother surfaces (\#) representing cells whereas the rough sections (\#\#) represent the assembled empty NPs. The element analysis highlighted this distribution since the sulfur element, characteristic of chondroitin sulfate present in the NP complex, is enhanced in the rough surface. PL-loaded hydrogels (Fig. 2D) are covered with a layer, rich in protein content as indicated by the high nitrogen content region identified in Fig. 2D by ${ }^{* *}$. The zone $\left(^{*}\right)$ represents a hole in that layer and the EDS analysis quantified the inner part of the material. The inner part shows a significant decrease in the nitrogen element (from 11.42 to 7.61 atomic percentage).

Fig. 3 (A-C) shows the results for the live/dead assay performed in the constructs one day post-encapsulation of hASCs. The assembled hydrogels presented a strong green fluorescent staining which indicates that the majority of the cells remained viable after the entrapment process. The hASCs entrapped in the hydrogel enriched with PLs presented a more elongated and less round shape, thus showing that the addition of lysate concentrate provides adhesion points to the embedded cells.

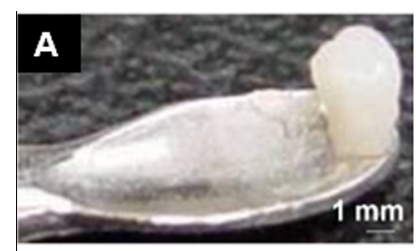

B

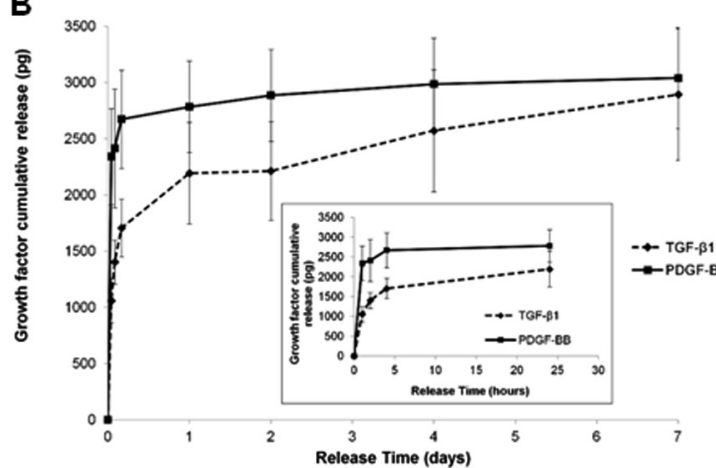

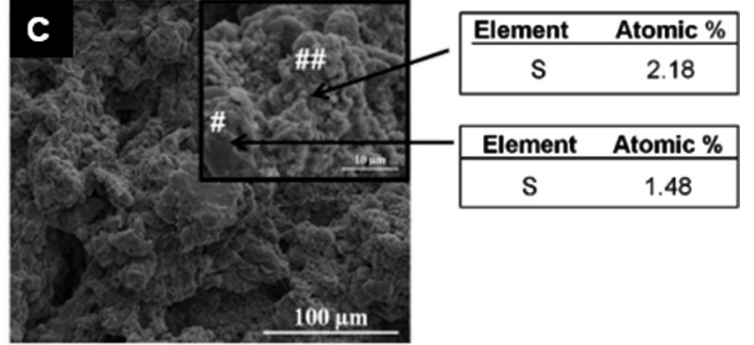

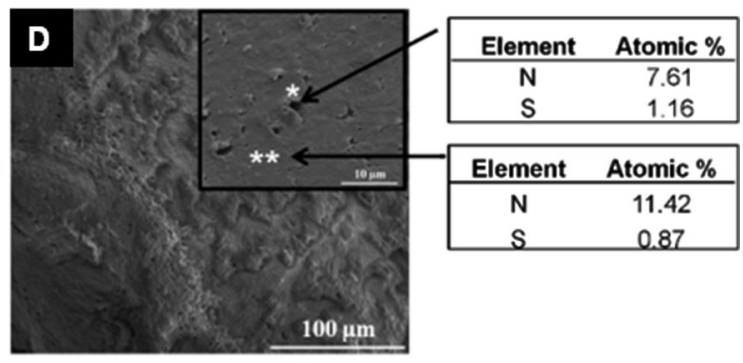

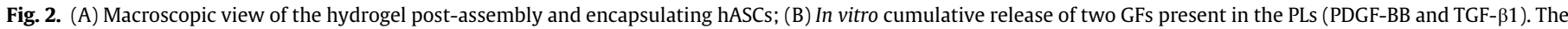

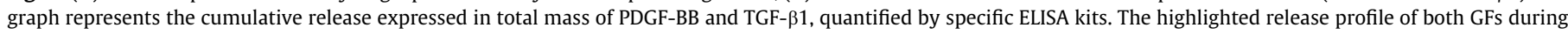

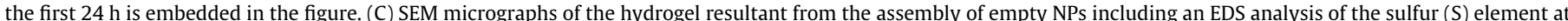

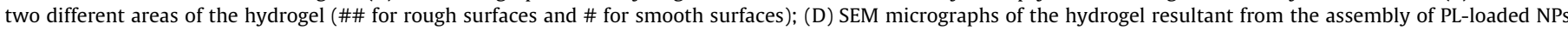

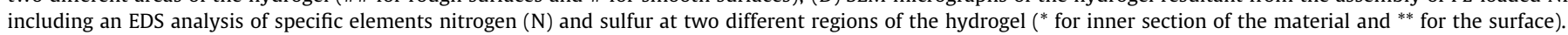



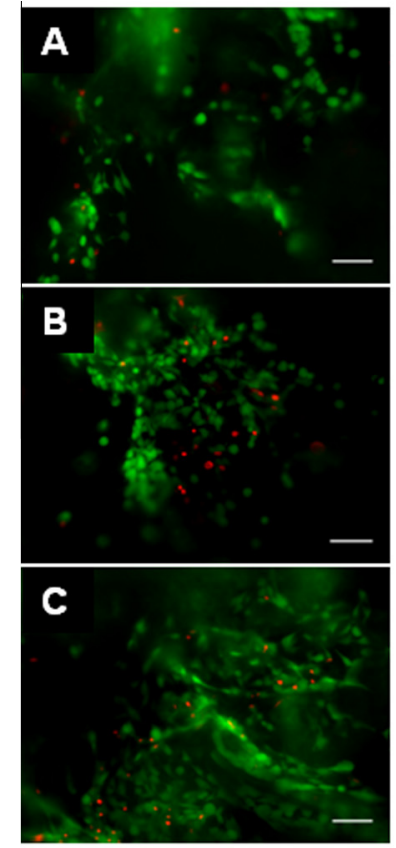

D

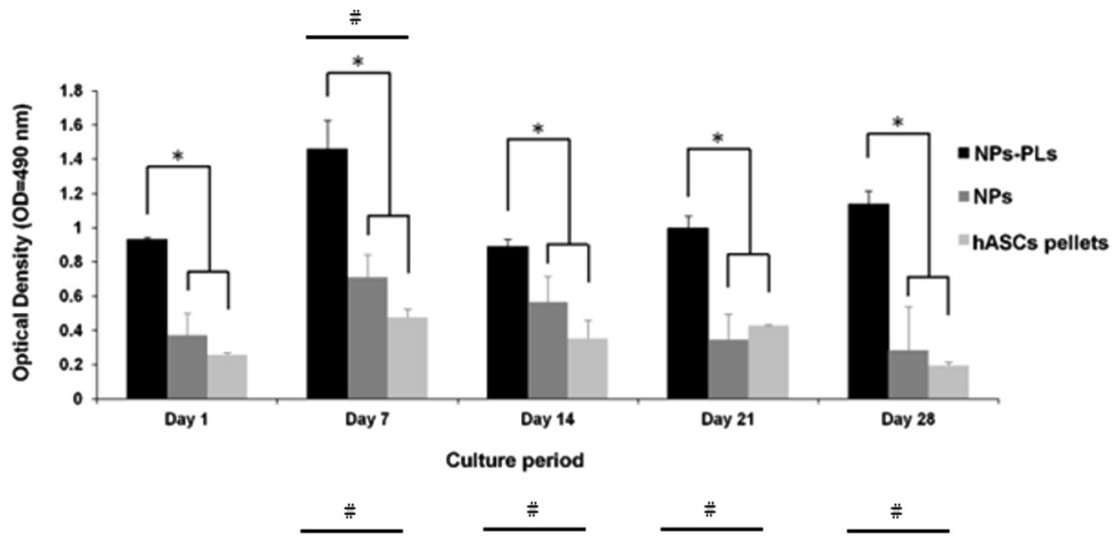

$\mathbf{E}$

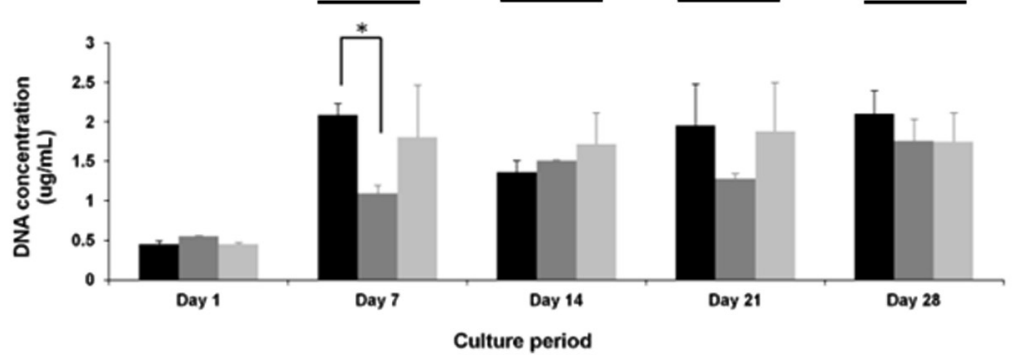

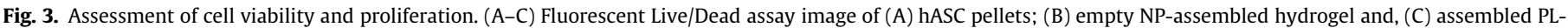

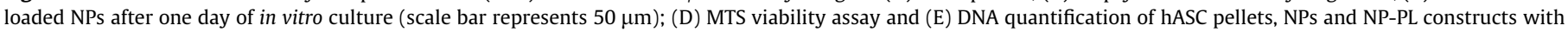

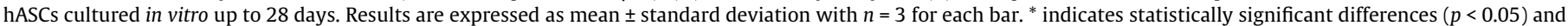
\# indicates statistically significant differences between the conditions at that specific culture period and day 1 of culture $(p<0.05)$.

Regarding the cell viability assessment by the MTS assay, the results obtained show that cells were able to remain viable in the constructs during the 28 days of experiment, although significant differences between the different formulations could be detected, as shown in Fig. 3D. After 7 days of culture, we could observe a statistically significant enhancement of total cell viability for all formulations. This may be consequence of two factors: either the cells were proliferating and thereby more MTS was metabolized or the proliferation rates were not that high but the mitochondrial machinery was highly active, converting higher amounts of MTS. Fig. 3E shows that after day 7, there was a significant increase in cell proliferation when compared to day 1. The micromass cultures of hASCs displayed a similar cell proliferation to the PL-loaded hydrogel at day 7, however their viability was significantly lower $(p<0.05)$. The viability of the hASCs entrapped in the PL-loaded $\mathrm{CH} / \mathrm{CS}$ assembled NP mesh was always higher in comparison with the controls despite the similar proliferation rate. Cell proliferation occurred mostly during the first week and remained relatively stable throughout the culture period (Fig. 3E).

In Fig. 4, the H\&E stained sections allowed the visualization of the cell distribution in the constructs. At early culture time points (e.g., day 7), cells at the peripheral region of the construct were flattened and aligned along the surface whereas cells exhibited a round morphology toward the center. While the pellet controls showed a homogeneous cell distribution during all culture periods, the hydrogels resultant from the assembly of NPs presented a progressive improvement in this specific feature. The intense red staining detected the hydrogel materials (PL-loaded or empty NPs), which was stronger at day 7 and 14 (Fig. 4B) than in the remaining time points because the NP core was still not strongly invaded by cells and newly deposited ECM. At the early stage of culture, the cells surrounded a core of NPs but their integration within the matrix progressed and increased with time.
PL-loaded hydrogel also presented a stronger interaction between cells and the materials in comparison with the hydrogel prepared with empty NPs (Fig. 4A and B). In fact, the hydrogel formed by the assembly of empty NPs gradually lost its stability, shape and dimensions, which led to cell loss from the construct (particularly after histological processing), as it is clear in Fig. 4B. On the contrary, a better integration of the cells in the PL-loaded hydrogel matrix was evident (Fig. 4A). On the other hand, the micromass culture presented the typical dense structure with cells embedded in a newly synthesized dense ECM (Fig. 4C). Although the initial cell number was the same for the control formulation, the hASC pellet sample size was always considerably smaller than the tested hydrogels and their size did not change drastically over time.

Fig. 5 depicts different read-outs for the assessment of chondrogenic differentiation of hASCs in each tested condition, as well as the deposition of cartilage-like ECM. The PL-loaded NP hydrogel resulted in a more homogeneous and intense alcian blue and safranin-O stainings (Fig. 5A) throughout the construct, with a more even distribution of cartilage ECM when compared with the empty NP assembled hydrogel (Fig. 5A). The hASC pellets present a more intense staining for both alcian blue and safranin-O (Fig. 5A) when compared with the other hydrogels, especially at day 14 . This intense staining can also be explained by the smaller size of the construct, and thus higher concentration of cells and ECM in a smaller area. In the case of the empty NP-assembled hydrogel, it is clear that the structure lost the stability with time, which led to cell loss and lower ECM formation, confirming the data obtained with H\&E staining.

The results previously presented in the histological analysis find some reflection on the Bern Score semi-quantitative analysis (Fig. 5B). Among the studied formulations, the control pellets exhibited the best results in terms of quality of the newly formed tissue. The scores at day 14 and 28 show a slightly positive 
hASCs+ PLs-NPs

A
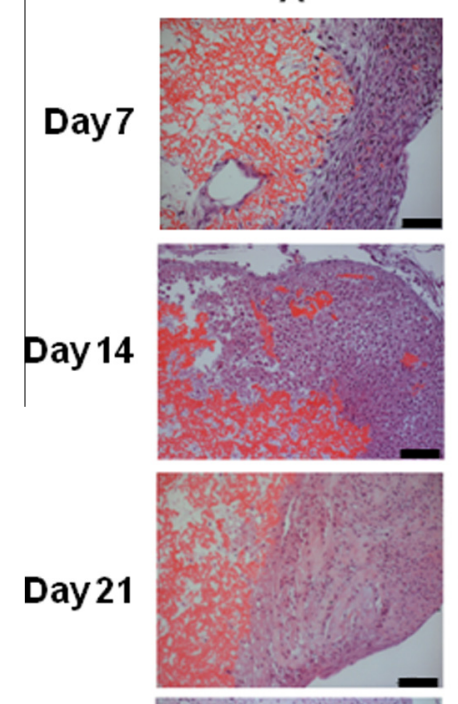

Day 28

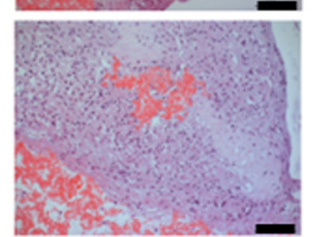

hASCs+ NPs

B
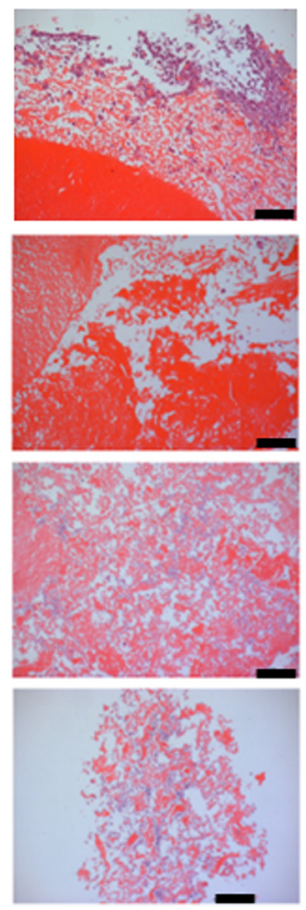

hASCs pellets

C
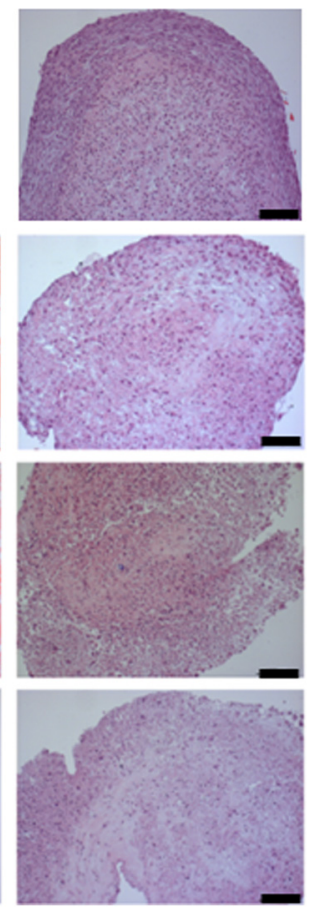

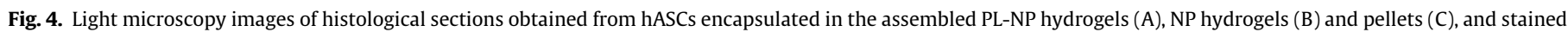
for $\mathrm{H} \& \mathrm{E}$, after 14, 21 and 28 days of culture in chondrogenic medium.

progression for the PL-loaded hydrogels. From day 14 to day 28, no relevant variations were encountered and despite the positive evaluation, these constructs were always ranked worse regarding the quality and performance of the newly formed tissue when compared to the pellet control. Pellet controls showed a slight decrease in the quality scores between days 14 and 28 of culture. In particular, scores for the micromass cultures peaked at day 14 , presenting statistically significant differences $(p<0.05)$ to all the remaining formulations and decreasing at day 28 . This can be explained by the material loss (both cellular and ECM) during the last 14 days of culture. The PL-loaded hydrogels always showed statistically significant differences $(p<0.05)$ to the assembled empty NP hydrogel, once again proving the importance of the adsorption of PLs to the NPs for the success of the developed construct.

The new ECM formation was also characterized by the immunolocalization of collagen type I and II and aggrecan as observed in Fig. 5A. Immunohistological analysis revealed the positive staining for both collagen type II and type I (Fig. 5A) in the newly formed cartilage-like matrix, for the PL-loaded hydrogel and the pellet control. Collagen II expression was particularly intense in the pellets, confirming the histological observation. Collagen II in the PL-loaded hydrogel was more clearly detected on the outer layers of the construct after 14 days of culture (Fig. 5A) and spread toward the core of the hydrogel until day 28. In Fig. $5 \mathrm{~A}$, we can also observe the accumulation of aggrecan mostly on the hASC pellets but also, although with less intensity, in the PL-loaded hydrogel. The hydrogels formed from the assembly of empty NPs only showed residual areas of collagen I and II (Fig. 5A) positive staining. The progressive cell loss from these non-loaded constructs is a limiting factor for the further success of these materials. For the empty NP hydrogel, the accumulation of aggrecan in the 3D structure was also reduced.
Due to the lack of gel stability and severe cell loss during the experiment, the evaluation of gene expression was not studied for the NP-assembled control.

The level of collagen type II expression for the PL-loaded NP hydrogel and for the hASCs pellets was upregulated throughout the experiment and it gradually increased over time, as it can be observed in Fig. 5C. The pellets always presented average higher upregulations of collagen type II when compared with the PLloaded hydrogel. However, no statistically significant difference $(p<0.05)$ between both study groups was detected. In the case of collagen type I expression (Fig. 5D), PL-enriched hydrogel expressed collagen I in higher levels than hASC pellets (with statistically significant differences $(p<0.05)$ ). Despite the stronger upregulation of collagen type I observed in the PL-loaded hydrogel, the upregulation level of the gene does not go further than the 3-fold increase, while for collagen type II expression, values reached around 40 -fold increase after 28 days of culture.

\section{Discussion}

Cartilage can be classified as a self-assembled tissue structure organized at different nano-, micro- and macro-scale levels [9]. The design criteria for the development of a bottom-up hydrogel must begin with proto-units of the nanomaterial assembled into higher order structures incorporating either chondrocytes or stem/progenitor cells, with appropriate signals for morphogenesis of cartilage [9].

The rationale behind this study (Fig. 1) followed a bottom-up approach as the hydrogels are developed from the assembly of NPs loaded with a protein cocktail that allows their stabilization and organization in a 3D structure, while it is simultaneously capable of entrapping hASCs. The process of tissue growth and development requires a constant supply of instructive cues and 
A

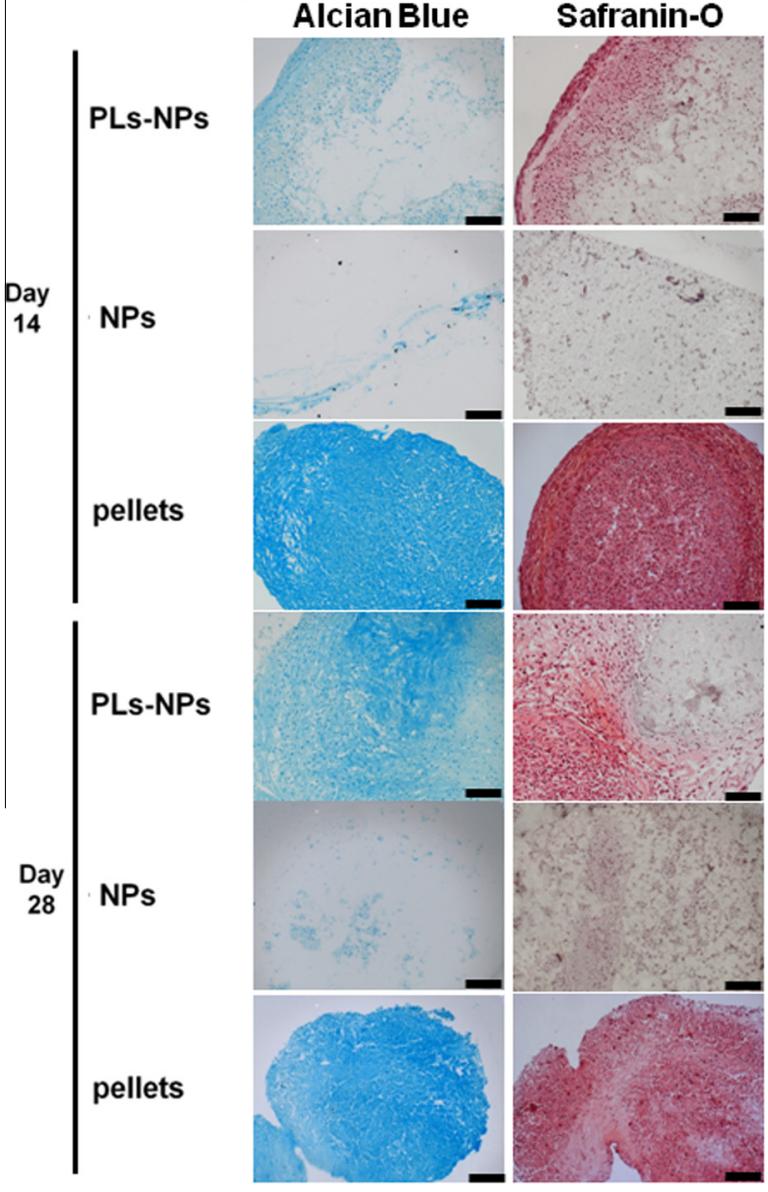

IMMUNOHISTOCHEMICAL CHARACTERIZATION

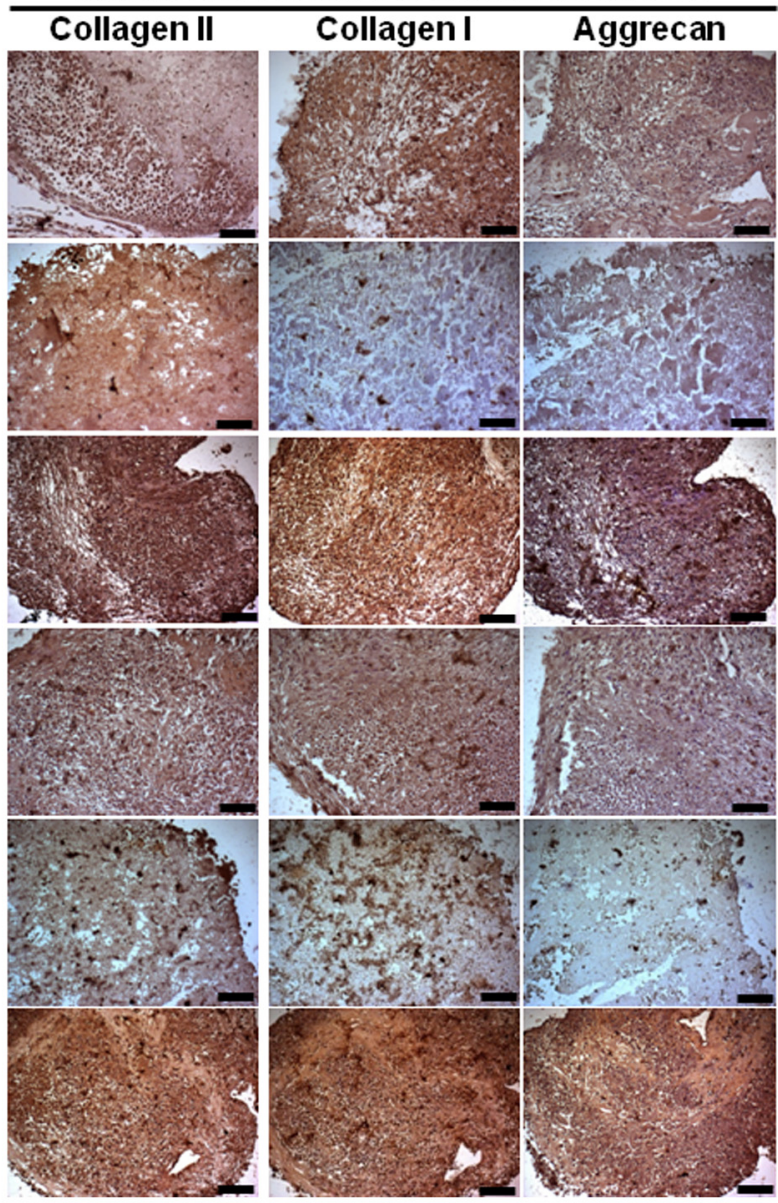

Collagen II

C

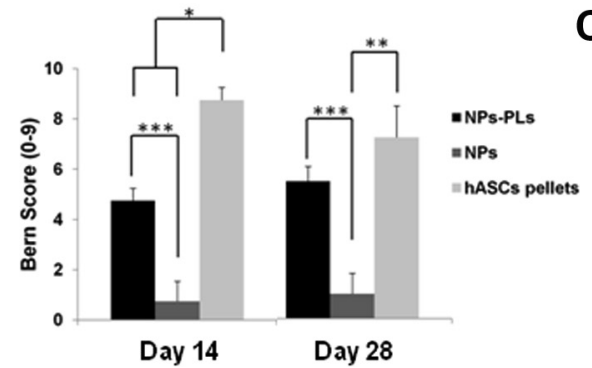

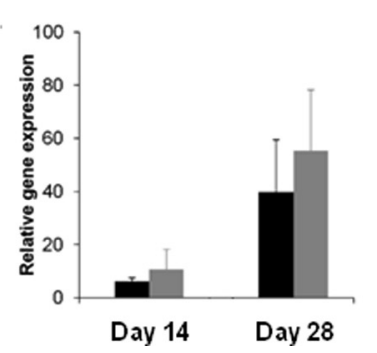

Collagen I

D

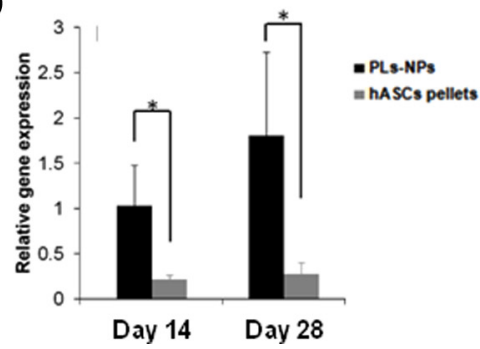

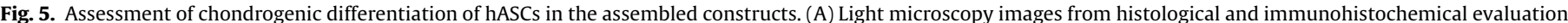

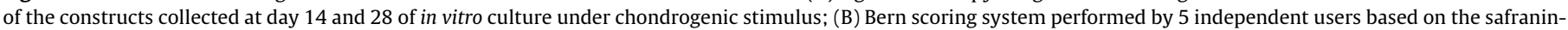

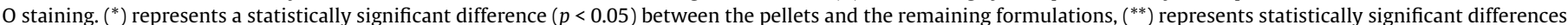

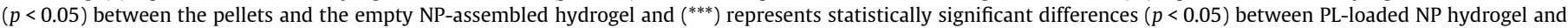

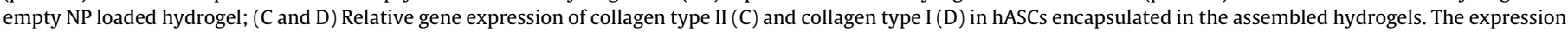

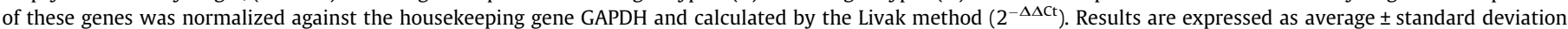
with $n=3$ for each bar. $\left(^{*}\right)$ indicates a statistically significant difference $(p<0.05)$ between the PL-loaded NP hydrogel and the hASC pellets.

in this strategy, these signaling molecules were provided by the GFs and bioactive proteins from PL concentrate (Fig. 1A). The polyelectrolyte complexation between two oppositely charged polysaccharides - chitosan and chondroitin sulfate, resulted in the formation of NPs, that besides the carrier and release function, are also able to induce the assembling of the NP-PLs into a 3D support for cartilage TE (Fig. 1B). In their native biological contexts, many polysaccharides are found organized at the nanoscale into complex assemblies such as the aggrecan aggregate in mammalian tissues [44]. Chitosan is the partially deacetylated form of chitin featuring a variable number of randomly located N-acetyl-glucosamine groups. These groups are also found in various GAGs present in articular cartilage, having specific interactions with many GFs, adhesion proteins and receptors [10]. Chondroitin sulfate is a sulfated GAG with reported beneficiary aspects in preventing the prevalence of osteoarthritis, namely on the prevention of proteoglycan degradation in the osteoarthritis model in rabbit and anti-inflammatory properties [10]. The presence of chondroitin sulfate has also been shown to enhance the expression of collagen type II in 3D cultures of chondrocytes [10] and to bind the 
important fibrillar forms of collagen I and II [44]. Due to the role of chitosan and chondroitin sulfate in cartilage tissue, the $\mathrm{CH} / \mathrm{CS}$ complex may exhibit beneficial features for cartilage regeneration and recreation of the environment of ECM and thus it seemed an appropriate selection for scaffolding material. Controlled GF delivery systems should be designed to deliver multiple soluble proteins at different rates to accelerate and enhance tissue regeneration $[30,45]$. These GFs can be immobilized in a matrix through physical adsorption (Fig. 1C), which involves the formation of ionic complexes or electrostatic interactions between oppositely charged groups on GFs and polymer chains on the substrate [46].

In this study, the cell-signaling molecules were incorporated into the bulk of the constructs and not only on the surface (Fig. 1D). The native ECM shows the ability to specifically store and sequester GFs and this feature has been emulated by this strategy [47]. In fact, the strategy herein described also promotes the positioning of functional motifs close to cell membrane receptors [48].

The observed burst release effect in the assembled construct (described in Fig. 2) was expected as the immobilization of proteins to the carriers through physical adsorption typically is usually characterized by less controlled release profiles [46]. However, in this strategy the lysates were used not only as bioactive agent source to promote cell proliferation and differentiation but also as a material to establish links between the NPs, in order to form the assembled hydrogel. The stability of the construct throughout the culture period suggests that the fast release of GFs loosely linked to the NPs during the first 7 days was not prolonged for the remaining culture period. The proteins and GFs present in the construct did not deadsorb as easily possibly because meanwhile hASCs also started to deposit cartilaginous ECM, creating a more compact and dense material.

The combination of results from viability and proliferation assessment suggests that cell pellets and PL-loaded hydrogels presented similar levels of cell proliferation, with the former displaying lower levels of cell viability. The availability of bioactive factors from PL dispersed in the hydrogel overcomes some of the diffusion issues associated with pellet cultures, therefore contributing for an overall higher cell viability of the hASCs entrapped in these constructs. Moreover, more than providing bioactive molecules for cell proliferation and differentiation, PLs also acted on the physical stabilization of the hydrogel, creating a more appropriate 3D environment for hASC survival.

PLs have also been indicated in the literature as inducers of cell proliferation but this fact was not evident in these studies $[33,49]$. However, as observed by the GF release profile, the delivery of bioactive agents occurred mostly during the first 7 days, more specifically at day 1 . The release profile matched the cell proliferation pattern because the latter was more evident precisely during the first 7 days of culture. The lack of significant cell proliferation in the remaining time points of the experiment might also indicate that hASCs were undergoing chondrogenic differentiation. The three formulations showed similar proliferation kinetics throughout most of the experiment. The similar cell proliferation profile exhibited by the PL-loaded NP hydrogel and the control pellet cultures might be explained by the structural environment in which the cells are cultured. In fact, even though when the cells are cultured in the presence of the PL-rich environment they have access to additional proteins, hASC pellets are exposed to the culture medium with a higher surface area and do not have a material barrier to delay their nutrient exchanges [29].

The H\&E stainings revealed that the cells entrapped in the hydrogel assembled from the PL-loaded NPs were progressively migrating toward the core of the hydrogel. PL-rich NPs acted as an attractant agent for the cells and the deadsorption of some of the PLs from the $\mathrm{CH} / \mathrm{CS}$ NPs led to bigger pores and gaps in the
3D structure, allowing cell infiltration and consequently better integration of the entrapped cells with the material. The constructs obtained from the empty NPs entrapping hASCs showed lower stability and the hydrogel disintegrated more easily, clearly demonstrating that the PLs are important not only for the release of GFs, but also for creating a stable 3D matrix.

Previous studies found in the literature concerning the use of hydrogels based only on PRP, mostly report the activation with bovine thrombin and crosslinking with calcium [50,51]. Despite the potential of those systems, the contraction observed in most of them during the culture period results in an ineffective strategy. The hydrogels quickly lose their shape and dimensions and consequently the construct disintegrates and becomes dispersed within the defect area. Herein, it is shown the multifunctional role of PLs, which enable the maintenance of the structure of the "biomaterial" through the combination of the lysates with a polysaccharidebased hydrogel, which resulted in a more stable formulation, closely mimicking the native ECM.

The results from cell proliferation and histological assessment suggest that the evolution of the tissue engineered cartilage constructs with culturing time progressed in two stages. The first one consisted of a cell growth phase characterized by increased cell proliferation until up to 7 days. The second stage can be described as a cell differentiation and tissue growth stage, which was characterized by decreased proliferation and increased collagen and proteoglycan deposition.

Histological score systems are frequently used to grade the healing process and tissue quality of cartilage defects. These semi-quantitative systems provide reliable information on the pathophysiological condition of the tissue being investigated and have good reproducibility. Among the various scoring systems proposed, the Bern score is considered to be one of the most reliable for evaluation of cartilage formation in vitro [52]. This semiquantitative analysis confirmed that the PL-loaded hydrogels contributed for the deposition of cartilage-like ECM and that their performance was statistically significantly improved when compared with the non-loaded NP-assembled hydrogel.

Collagen type II is responsible for the tensile properties of cartilage tissue, forming a dense network of fibers responsible for retaining the aggrecan during compressive loading [7] and it is the most important protein produced by chondrocytes. Although collagen type I is not found in mature hyaline cartilage ECM, it may play vital roles regulating both in mesenchymal condensation and chondrogenic differentiation [7]. Mesenchymal progenitor cells can secrete an ECM rich in collagen type I before the onset of overt chondrogenesis. However, as overt chondrogenesis begins and the expression of mature cartilage matrix molecules starts, the production of collagen type I stops [7]. Both collagen II and I were upregulated for the PL-loaded hydrogel, however the enhanced expression was significantly higher for collagen II, thus suggesting the formation of new hyaline-type cartilage tissue rather than fibrous cartilage. The differentiation of hASCs toward the chondrogenic phenotype was an ongoing process and a complete mature chondrocyte phenotype had not yet been settled. Both the histological and gene expression confirmed that chondrogenic differentiation was higher for the hASC pellets positive controls, which can be explained by the supplementation with TGF- $\beta 1$ in the culture medium.

In this study, the role of PLs as a source of bioactive agents to promote enhancement of chondrogenic differentiation was limited. Although PLs contain TGF- $\beta 1$, that plays a key role in the regulation of cellular proliferation, differentiation and cartilage matrix formation $[4,8,25,27]$ and can exhibit differential effects depending on the differentiation stage of the target cells [53], the chondrogenic differentiation was not clearly enhanced when compared with the control formulation. These findings might be explained 
by the quick GF release profile during the cell proliferation stage and by the initial loading of TGF- $\beta 1$ in the PL-loaded hydrogels to be approximately $4 \mathrm{ng}$ per construct. This value is well below the standardized TGF- $\beta 1$-supplemented chondrogenic medium used for the pellet cultures in our positive controls.

On the other hand, the structural role of the lysates as a decisive component of this "bottom-up" design of the hydrogel is the major outcome of this strategy. PLs acted as a raw material to promote the connections and links to the NPs, in order to fabricate a hydrogel mesh for the entrapment of stem cells that will be able to better withstand the in vivo environment conditions than the hydrogel resultant from the assembly of empty NPs or the cell pellets. The design of the system aims for an injectable minimally invasive approach, providing an advantage over the injection of cell suspensions, which lack structural support and will eventually diffuse out of the injection site. The combination of PL-loaded NPs with hASCs will avoid the migration of cells toward undesired locations while simultaneously acquiring the shape of the defect and providing an additional template for mechanical support at the injection site. Moreover, the dynamic interplay between cells and the cartilage ECM molecules, such as chondroitin sulfate, is a contributing factor to the beneficial effect of the ECM-mimicking hydrogel that we propose in this study, since the environmental context is very similar to the native cartilage tissue [54].

\section{Conclusion}

This study showed that it was possible to use PLs simultaneously as a source and carrier of GFs, assembling both functionalities into a 3D structural support through a "bottomup" approach. This approach uses the assembly of NPs, promoted by the adsorption of PLs to the surface of the NPs and allowed overcoming the usual contraction effect found in PL based hydrogels. Furthermore, the proposed methodology enabled the development of an innovative system that provides an adequate environment for the encapsulation of hASCs, through the enrichment with the GFs released from the PLs.

Furthermore, the use of the complex $\mathrm{CH} / \mathrm{CS}$ as one of the components of the matrix, in the NP formulations, is also appropriate for the development of constructs for cartilage regeneration because the interactions between chondroitin sulfate, one of the most important GAGs in ECM, and the proteins, present in the PLs cocktail, mimic the in situ environment of cartilage ECM.

In summary, this study demonstrated that PLs can work not only as a GF supplement for cell proliferation and differentiation but can also provide stability of a support structure, through the entanglement of the protein chains with the $\mathrm{CH} / \mathrm{CS}$ NPs, assembled into a 3D hydrogel. It is envisioned the translation of this system into the clinic by the isolation of autologous blood in-theater with follow-up combination with hASCs from the same patient and $\mathrm{CH} /$ CS NPs to produce and injectable biomaterial for cartilage regeneration.

\section{Acknowledgments}

The authors thank Fundação para a Ciência e Tecnologia for V.E. Santo and E.G. Popa's PhD grants (SFRH/BD/39486/2007 and SFRH/ $\mathrm{BD} / 64070 / 2009$, respectively). This work was carried out under the scope of the European NoE EXPERTISSUES (NMP3-CT-2004500283) and it was partially supported by the European FP7 Project Find and Bind (NMP4-SL-2009-229292). We thank IPS for providing the human platelet donations and Hospital da Prelada for the human adipose tissue samples.

\section{Appendix A. Figures with essential colour discrimination}

Certain figures in this article, particularly Figs. 1-5 are difficult to interpret in black and white. The full colour images can be found in the on-line version, at http://dx.doi.org/10.1016/j.actbio.2015. 03.015.

\section{Appendix B. Supplementary data}

Supplementary data associated with this article can be found, in the online version, at http://dx.doi.org/10.1016/j.actbio.2015.03. 015 .

\section{References}

[1] Place ES, Evans ND, Stevens MM. Complexity in biomaterials for tissue engineering. Nat Mater 2009;8(6):457-70.

[2] Chung C, Burdick JA. Engineering cartilage tissue. Adv Drug Deliv Rev 2008;60(2):243-62.

[3] Hildner F, Albrecht C, Gabriel C, Redl H, van Griensven M. State of the art and future perspectives of articular cartilage regeneration: a focus on adiposedderived stem cells and platelet-derived products. J Tissue Eng Regen Med 2011;5(4):e36-51.

[4] Lee SH, Shin H. Matrices and scaffolds for delivery of bioactive molecules in bone and cartilage tissue engineering. Adv Drug Deliv Rev 2007;59(45):339-59.

[5] Mano JF, Reis RL. Osteochondral defects: present situation and tissue engineering approaches. J Tissue Eng Regen Med 2007;1(4):261-73.

[6] Diekman BO, Rowland CR, Lennon DP, Caplan AI, Guilak F. Chondrogenesis of adult stem cells from adipose tissue and bone marrow: induction by growth factors and cartilage-derived matrix. Tissue Eng Part A 2010;16(2):523-33.

[7] Bobick BE, Chen FH, Le AM, Tuan RS. Regulation of the chondrogenic phenotype in culture. Birth Defects Res Part C: Embryo Today 2009;87(4):351-71.

[8] Sohier J, Moroni L, van Blitterswijk C, de Groot K, Bezemer JM. Critical factors in the design of growth factor releasing scaffolds for cartilage tissue engineering. Expert Opin Drug Deliv 2008;5(5):543-66.

[9] Reddi AH, Becerra J, Andrades JA. Nanomaterials and hydrogel scaffolds for articular cartilage regeneration. Tissue Eng Part B Rev 2011;17(5):301-5.

[10] Balakrishnan B, Banerjee R. Biopolymer-based hydrogels for cartilage tissue engineering. Chem Rev 2011;111(8):4453-74.

[11] Wang CC, Yang KC, Lin KH, Liu HC, Lin FH. A highly organized threedimensional alginate scaffold for cartilage tissue engineering prepared by microfluidic technology. Biomaterials 2011;32(29):7118-26.

[12] Jeon O, Powell C, Ahmed SM, Alsberg E. Biodegradable, photocrosslinked alginate hydrogels with independently tailorable physical properties and cell adhesivity. Tissue Eng Part A 2010;16(9):2915-25.

[13] Sheehy EJ, Buckley CT, Kelly DJ. Chondrocytes and bone marrow-derived mesenchymal stem cells undergoing chondrogenesis in agarose hydrogels of solid and channelled architectures respond differentially to dynamic culture conditions. J Tissue Eng Regen Med 2011;5(9):747-58.

[14] Mauck RL, Yuan X, Tuan RS. Chondrogenic differentiation and functional maturation of bovine mesenchymal stem cells in long-term agarose culture. Osteoarthritis Cartilage 2006;14(2):179-89.

[15] Lien SM, Ko LY, Huang TJ. Effect of pore size on ECM secretion and cell growth in gelatin scaffold for articular cartilage tissue engineering. Acta Biomater 2009;5(2):670-9.

[16] Kim IL, Mauck RL, Burdick JA. Hydrogel design for cartilage tissue engineering: a case study with hyaluronic acid. Biomaterials 2011;32(34):8771-82.

[17] Toh WS, Lee EH, Guo XM, Chan JKY, Yeow CH, Choo AB, et al. Cartilage repair using hyaluronan hydrogel-encapsulated human embryonic stem cell-derived chondrogenic cells. Biomaterials 2010;31(27):6968-80.

[18] Oliveira JT, Santos TC, Martins L, Picciochi R, Marques AP, Castro AG, et al. Gellan gum injectable hydrogels for cartilage tissue engineering applications: in vitro studies and preliminary in vivo evaluation. Tissue Eng Part A 2009;16(1):343-53.

[19] Oliveira JT, Santos TC, Martins L, Silva MA, Marques AP, Castro AG, et al. Performance of new gellan gum hydrogels combined with human articular chondrocytes for cartilage regeneration when subcutaneously implanted in nude mice. J Tissue Eng Regen Med 2009;3(7):493-500.

[20] Rocha PM, Santo VE, Gomes ME, Reis RL, Mano JF. Encapsulation of adiposederived stem cells and transforming growth factor- $\beta 1$ in carrageenan-based hydrogels for cartilage tissue engineering. J Bioact Compat Pol 2011;26(5):493-507.

[21] Popa EG, Gomes ME, Reis RL. Cell delivery systems using alginate-carrageenan hydrogel beads and fibers for regenerative medicine applications. Biomacromolecules 2012;12(11):3952-61.

[22] Sage A, Chang AA, Schumacher BL, Sah RL, Watson D. Cartilage outgrowth in fibrin scaffolds. Am J Rhinol Allergy 2009;23(5):486-91.

[23] Ahmed TAE, Dare EV, Hincke M. Fibrin: a versatile scaffold for tissue engineering applications. Tissue Eng Part B Rev 2008;14(2):199-215. 
[24] Hwang N, Im S, Wu P, Bichara D, Zhao X, Randolph M, et al. Chondrogenic priming adipose-mesenchymal stem cells for cartilage tissue regeneration. Pharm Res 2011;28(6):1395-405.

[25] Cancedda R, Dozin B, Giannoni P, Quarto R. Tissue engineering and cell therapy of cartilage and bone. Matrix Biol 2003;22(1):81-91.

[26] Kim HJ, Im GI. Chondrogenic differentiation of adipose tissue-derived mesenchymal stem cells: greater doses of growth factor are necessary. J Orthop Res 2009;27(5):612-9.

[27] Lin $Y$ et al. Molecular and cellular characterization during chondrogenic differentiation of adipose tissue-derived stromal cells in vitro and cartilage formation in vivo. J Cell Mol Med 2005;9(4):929-39.

[28] Awad HA, Quinn Wickham M, Leddy HA, Gimble JM, Guilak F. Chondrogenic differentiation of adipose-derived adult stem cells in agarose, alginate, and gelatin scaffolds. Biomaterials 2004;25(16):3211-22.

[29] Yoon IS et al. Proliferation and chondrogenic differentiation of human adiposederived mesenchymal stem cells in porous hyaluronic acid scaffold. J Biosci Bioeng 2011;112(4):402-8.

[30] Chen FM, Zhang M, Wu ZF. Towards delivery of multiple growth factors in tissue engineering. Biomaterials 2010;31(24):6279-308.

[31] Dolder JVD, Mooren R, Vloon APG, Stoelinga PJW, Jansen JA. Platelet-rich plasma: quantification of growth factor levels and the effect on growth and differentiation of rat bone marrow cells. Tissue Eng 2006;12(11):3067-73.

[32] Soomekh DJ. Current concepts for the use of platelet-rich plasma in the foot and ankle. Clin Podiatr Med Surg 2011;28(1):155-70.

[33] Akeda $\mathrm{K}$ et al. Platelet-rich plasma stimulates porcine articular chondrocyte proliferation and matrix biosynthesis. Osteoarthritis Cartilage 2006;14(12): $1272-80$.

[34] Spreafico A et al. Biochemical investigation of the effects of human platelet releasates on human articular chondrocytes. J Cell Biochem 2009;108(5): 1153-65.

[35] Mishra A, Tummala P, King A, Lee B, Kraus M, Tse V, et al. Buffered platelet-rich plasma enhances mesenchymal stem cell proliferation and chondrogenic differentiation. Tissue Eng Part C Methods 2009;15(3):431-5.

[36] Custódio CA, Santo VE, Oliveira MB, Gomes ME, Reis RL, Mano JF. Functionalized microparticles producing scaffolds in combination with cells. Adv Funct Mater 2014;24(10):1391-400.

[37] Steward A, Liu Y, Wagner D. Engineering cell attachments to scaffolds in cartilage tissue engineering. JOM J Min Met Mat S 2011;63(4):74-82.

[38] Santo VE, Gomes ME, Mano JF, Reis RL. Chitosan-chondroitin sulphate nanoparticles for controlled delivery of platelet lysates in bone regenerative medicine. J Tissue Eng Regen Med 2012;6(Suppl 3):s47-59.
[39] Zaky SH, Ottonello A, Strada P, Cancedda R, Mastrogiacomo M. Platelet lysate favours in vitro expansion of human bone marrow stromal cells for bone and cartilage engineering. J Tissue Eng Regen Med 2008;2(8):472-81.

[40] Rada T, Reis RL, Gomes ME. Distinct stem cells subpopulations isolated from human adipose tissue exhibit different chondrogenic and osteogenic differentiation potential. Stem Cell Rev 2011;7(1):64-76.

[41] Rada T, Reis RL, Gomes ME. Novel method for the isolation of adipose stem cells (ASCs). J Tissue Eng Regen Med 2009;3(2):158-9.

[42] Grogan SP et al. Visual histological grading system for the evaluation of in vitro-generated neocartilage. Tissue Eng 2006;12(8):2141-9.

[43] Livak KJ, Schmittgen TD. Analysis of relative gene expression data using realtime quantitative PCR and the 2(-Delta Delta C(T)) method. Methods 2001;25(4):402-8

[44] Boddohi S, Kipper MJ. Engineering nanoassemblies of polysaccharides. Adv Mater 2010;22(28):2998-3016.

[45] Santo VE, Gomes ME, Mano JF, Reis RL. Controlled release strategies for bone, cartilage, and osteochondral engineering - Part II: challenges on the evolution from single to multiple bioactive factor delivery. Tissue Eng Part B Rev 2013:19(4):327-52.

[46] Tayalia P, Mooney DJ. Controlled growth factor delivery for tissue engineering. Adv Mater 2009;21(32-33):3269-85.

[47] von der Mark K, Park J, Bauer S, Schmuki P. Nanoscale engineering of biomimetic surfaces: cues from the extracellular matrix. Cell Tissue Res 2010;339(1):131-53.

[48] Gelain F. Novel opportunities and challenges offered by nanobiomaterials in tissue engineering. Int J Nanomedicine 2008;3(4):415-24.

[49] Lucarelli E et al. Platelet-derived growth factors enhance proliferation of human stromal stem cells. Biomaterials 2003;24(18):3095-100.

[50] Butterfield KJ, Bennett J, Gronowicz G, Adams A. Effect of platelet-rich plasma with autogenous bone graft for maxillary sinus augmentation in a rabbit model. J Oral Maxillofac Surg 2005;63(3):370-6.

[51] Hokugo A et al. Controlled release of platelet growth factors enhances bone regeneration ar rabbit calvaria. Oral Surg Oral Med O 2007;104(1):44-8.

[52] Rutgers M, van Pelt MJP, Dhert WJA, Creemers LB, Saris DBF. Evaluation of histological scoring systems for tissue-engineered, repaired and osteoarthritic cartilage. Osteoarthr Cartilage 2010;18(1):12-23.

[53] Csaki C, Schneider PRA, Shakibaei M. Mesenchymal stem cells as a potential pool for cartilage tissue engineering. Ann Anat 2008;190(5):395-412.

[54] Choi KH, Choi BH, Park SR, Kim BJ, Min BH. The chondrogenic differentiation of mesenchymal stem cells on an extracellular matrix scaffold derived from porcine chondrocytes. Biomaterials 2010;31(20):5355-65. 